\title{
Validación del cuestionario de autoevaluación para unidades administrativas y de servicios
}

\author{
Validation of the self assessment survey of the administrative and service units
}

\author{
M. Soledad Campo Herrera ${ }^{1 *} \quad$ Ma. Carmen Ortega Navas ${ }^{2}$ \\ Recibido 22 de enero de 2016, aceptado 14 de julio de 2016 \\ Received: January 22, 2016 Accepted: July 14, 2016
}

\begin{abstract}
RESUMEN
Por más de una década la Universidad Mayor (UM) ha realizado diversos procesos de autoevaluación y acreditación, que han comprometido tanto a la institución, como a un número importante de carreras y programas. Esto ha significado, entre otras cosas, la aplicación anual de diferentes encuestas de satisfacción institucional con la participación de diferentes actores (personal no académico), aportando información sobre el desempeño de las unidades administrativas y de gestión.

En este artículo se presentan los resultados de la validación del Cuestionario de Autoevaluación de la Universidad Mayor, instrumento organizado sobre la base de los criterios del modelo European Foundation of Quality Management (EFQM).

La investigación se incluye dentro de los estudios exploratorios y descriptivos. La validación de este instrumento se realizó en dos etapas. En la primera, se hizo un mejoramiento progresivo mediante la consulta a expertos de los distintos estamentos del personal involucrado en esta evaluación. En una segunda, se determinó la tendencia y regularidad del instrumento al aplicarlo a una muestra de 397 personas, de 36 unidades diferentes de la misma organización. Finalmente, los resultados indican que el instrumento mide de una forma válida y precisa las dimensiones evaluadas.
\end{abstract}

Palabras clave: Calidad, autoevaluación, unidades administrativas y de servicios, instrumento de evaluación, validación.

\section{ABSTRACT}

For over than a decade Universidad Mayor (UM) has been implementing various accreditation and self-study assessment processes involving the institution as a whole, as well as a considerable number of academic programs. As a consequence, there have been yearly institutional satisfaction surveys answered by teachers and students that provided information on different academic and management units, making evident the need to seek the opinion of the administrative staff as well.

This paper presents the results of the validation of the self-assessment questionnaire devised for the administrative and service units of the UM, instrument organized according to the criteria of the European Foundation of Quality Management (EFQM) model.

The research is considered within the exploratory and descriptive studies. The validation of this instrument was carried out in two stages, firstly, by consulting the opinions of experts and different representatives of

1 Universidad Mayor. Vicerrectoría Académica y de Aseguramiento de la Calidad. San Pío X 2422. Santiago, Chile. E-mail: soledad.campo@umayor.cl

2 Universidad Nacional de Educación a Distancia, UNED. Departamento de Teoría de la Educación y Pedagogía Social. C/ Juan del Rosal. 14. 28040. Madrid, España. E-mail: cortega@edu.uned.es

* Autor de correspondencia 
the staff, which resulted in a progressive improvement of the tool, secondly, by applying the instrument to a representative population sample of 397 people from 36 different units of the institution which validated the regularity and tendency of the instrument. Finally, the results show that the instrument is reliable and valid to measure the dimensions and variables.

Keywords: Quality, self-study assessment, administrative staff, evaluation tools and validation.

\section{INTRODUCCIÓN}

En Chile se tiene aún poca experiencia en realizar autoevaluación en el área académica, lo que hace que este aún más distante, la autoevaluación en unidades administrativas o de servicios. Cualquier iniciativa de autoevaluación requiere por una parte de personal calificado que apoye los procesos al interior de las instituciones, como de procedimientos y mecanismos que favorezcan los procesos internos, siendo la autoevaluación una de ellas, aunque estos mecanismos y procedimientos aún son poco difundidos en nuestro país para este tipo de unidades.

Hax y Ugarte [1] señalan que "No existe un modelo universal ni tampoco un concepto único sobre el sentido último de la actividad universitaria, que pueda ser trasplantado de un país a otro, como tampoco resultaría legítimo aspirar a llegar a un tipo de respuesta hegemónica dentro de un mismo país".

Al no existir un único modelo de universidad como tampoco una única manera de realizar una autoevaluación en un grupo de actores claves para asegurar la calidad del servicio que se brinda a los estudiantes, se indagó, para detectar qué se utilizaba con fines similares a lo que se requería, que fuera cercano y pudiera aportar, aunque fuera en parte, a resolver esta necesidad. Es así que se llegó a la Fundación Europea para la Gestión de la Calidad (European Foundation for Quality Management), cuya sigla en inglés es EFQM. Esta institución, sin fines de lucro, cuenta con una metodología denominada "Modelo Europeo para la Gestión de la Calidad Total" o como aparece en algunas traducciones, también se le denomina "Modelo EFQM de Calidad y Excelencia", cuyo propósito es favorecer procesos de autoevaluación y definición de procesos de mejora continua, tanto en unidades o contextos organizacionales de empresas públicas y privadas. Su metodología es muy similar a otras utilizadas por ejemplo por Japón (Premio Deming) para otorgar el premio nacional a la calidad y a la de Estados Unidos para fines similares (Premio Malcom Baldrige) [2]. Si bien no siempre se pueden aplicar como originalmente fueron concebidas, permiten orientar a las organizaciones que buscan el mejoramiento de la calidad, puesto que entregan conceptos, precisiones técnicas o criterios, por nombrar algunos elementos, que permiten orientar procesos de esta naturaleza.

Es importante señalar que a fines de la década de 1990 la Fundación Iberoamericana para la Gestión de la Calidad (FUNDIBQ) aprobó un modelo para Iberoamérica, similar al original, pero aprovechando la experiencia que brindaba la ejecución del modelo EFQM y los aspectos que se visualizaba debían mejorarse. Este modelo iberoamericano se adaptó de manera que las instituciones universitarias pudieran utilizarlo, así como establecimientos educacionales de educación básica y media [2].

Otro desarrollo de este modelo se encuentra en España, puesto que el Ministerio de Educación, Cultura y Deporte por medio de su Secretaría Técnica elaboró una serie de indicadores que permiten guiar la autoevaluación en unidades de servicio universitario [2]. La autoevaluación basada en los modelos centrados en la búsqueda de la calidad, pretenden fundamentalmente definir los aspectos considerados fortalezas y debilidades para que, desde estos, se determinen áreas que requieren intervención y reconocer aspectos posibles de desarrollar, diseñando planes de mejora que orienten la gestión de la unidad como resultado de la evaluación [3]. Esto es importante para proveer del "Mejor Servicio" en el más amplio sentido, de forma que la universidad se posesione de manera estratégica siendo eficiente y efectiva en su propósito central, que es formar profesionales, diferenciándose en la provisión de servicios para atraer los mejores talentos a sus aulas [1].

Sin embargo, la literatura especializada reporta que la implementación de estrategias que implican el aseguramiento de la calidad, requiere de cambios 
culturales, como lo es trabajar para la excelencia de la calidad, requieren de ir acompañados de capacitación para el personal, recursos, tiempo y compromiso de las altas autoridades, entre otros elementos. Si a esto se adiciona que la institución cuenta con un personal heterogéneo en cuanto a formación, proyección laboral, años de experiencia, entonces la tarea de la implementación es aún más desafiante. En este contexto, el modelo por si solo es insuficiente y puede llegar a ser inefectivo [1,3].

Uno de los problemas detectados en los procesos de autoevaluación para enfrentar procesos de acreditación nacional e internacional, es que la mirada generalmente se centra en los resultados académicos o lo que está directamente en su entorno, como condiciones de operación conducente para determinar la calidad $\mathrm{y}$, en menor medida, se consideran las unidades administrativas y de servicios como actores claves de consulta. Muchas veces parece obvio que estas últimas deben funcionar en torno al quehacer académico, pero no siempre se producen las sinergias necesarias que aseguren la complementariedad requerida $[3,5]$.

Una muestra de lo señalado, son las propuestas de cuestionarios de la Comisión Nacional de Acreditación (CNA-Chile) para apoyar los procesos de autoevaluación de las instituciones y programas del país, los que se centran en los estudiantes, docentes, titulados y empleadores, puesto que el fin de estos es recoger percepciones para servir de insumo a los procesos más que en brindar apoyo a los procedimientos internos de una universidad.

Los que se benefician de las unidades, estudiantes y profesores, principalmente, sean estas administrativas o de servicios con que cuenta la universidad, pueden valorar, expresando su grado de satisfacción por medio de encuestas o cuestionarios que son administrados por la propia institución. Pero también, se pueden recoger las percepciones del personal administrativo o profesionales que están trabajando en las unidades, para evaluar los servicios que brindan y de esta forma, de manera autocrítica, dar el espacio para proponer mejoras tendientes a satisfacer a sus usuarios y mejorar la calidad de lo que realizan.

Al no existir un sistema probado como tampoco un instrumento que respondiera a la necesidad, se realizó la investigación para determinar las características de dicho sistema y se tomó la decisión a partir del primer diagnóstico que era fundamental contar con un instrumento que posibilite identificar las principales brechas y las fortalezas, desde la perspectiva del estamento de funcionarios. Los que normalmente eran espectadores de los procesos de autoevaluación.

El primer paso entonces, fue elaborar el instrumento que permitió recoger las percepciones de los diferentes funcionarios que laboran en las unidades de servicios y administrativas para facilitar la autoevaluación de las unidades y favorecer la identificación del grado de calidad de la unidad respectiva. Ratifica este paso, que diferentes autores indican que en el contexto de la autoevaluación y explícitamente en la aplicación del Modelo EFQM, uno de los métodos más utilizados es la aplicación de un cuestionario. Sin embargo, no se encontró ninguno que fuera útil para las características de una institución como la UM. Especialmente porque cualquier propuesta de esta naturaleza requiere de la participación de las personas involucradas de modo de concurrir como corresponsable de los resultados de los cambios (eficacia), mejorando para producir cambios y aprovechar potenciando los recursos (eficiencia) [4].

Los resultados de este trabajo de investigación permitieron verificar la confiabilidad de la encuesta y validar sus supuestos en el contexto de la Universidad Mayor de Chile.

\section{ANÁLISIS TEÓRICO}

\section{Antecedentes}

Durante los últimos 25 años Chile ha experimentado un desarrollo importante en los procesos de aseguramiento de la calidad, donde se ha destacado en las instituciones oficinas especializadas que apoyan dichos procesos. Esta profesionalización acompañada de una serie de regulaciones externas ha determinado que se vayan abriendo nuevas posibilidades en cuanto a realizar desarrollo teóricos y prácticos para mejorar las prácticas en la búsqueda de la calidad [5].

Haciendo un poco de historia, merece la pena revisar una cronología breve de cómo la calidad ha pasado a permear diferentes ámbitos, que competen al mundo de la gestión en las organizaciones y que ha pasado progresivamente a ser un tema central en otras áreas como lo es la educación universitaria, puesto que es una necesidad de cambio desde la universidad concebida de manera tradicional a la del actual 
siglo, que conlleva exigencias diferentes tanto en la formación, las relaciones internas y externas de las instituciones como un sinfín de funciones que también han sufrido la transformación [4-5].

Un primer aspecto que se debe considerar es lo que se denomina como "Calidad Total", proceso que ha tenido transformaciones en el tiempo, puesto que a partir de los años 1980 las tendencias en la gestión de calidad, se concentraron principalmente en dos tipos de modelos: el de las normas ISO 9.000 y el Modelo de Excelencia Empresarial (EFQ), entendiendo que existen también otros como el Modelo de Malcolm Baldrige de Norteamérica, los Manuales de Buenas Prácticas muy difundidos en América Latina, por nombrar algunos [6]. En el tiempo se fue pasando desde el control de la calidad, la garantía de la misma hasta llegar a la calidad total que no es otra cosa que observar el sistema de gestión organizacional que incluya el control y la garantía, es decir, las diferentes dimensiones implicadas. Suárez se refiere a la Calidad Total y plantea que la "Calidad debe ser hecha, no por inspección sino por construcción en todos los puntos del proceso de trabajo..." [7].

Estos sistemas cuentan con una serie de principios que son aplicables a las instituciones universitarias, como por ejemplo $[1,9]$.

- Lograr la satisfacción de necesidades y expectativas de las personas que demandan el servicio, que en este caso son los estudiantes, sus familias principalmente, además del sistema social que requiere de profesionales bien formados.

- Desarrollar procesos de mejora continua en los procesos y en las actividades. Se trata de realizar assessment permanente.

- Liderazgo y compromiso de la dirección. En el caso de la universidad, es el gobierno y sus autoridades quienes se deben comprometer con la calidad.

- Participación y trabajo en equipo de las personas que trabajan en la organización. Sin la participación no se construye una comunidad universitaria, por tanto, este aspecto es fundamental.

- Identificación y gestión de los procesos claves de la organización. Los procesos claves aportan a conseguir los resultados, constituyéndose en un aspecto central para asegurar la efectividad de la universidad.
- Toma de decisiones basada en datos y hechos objetivos sobre la gestión. Mediante la introducción de ajustes y/o mejoras en los momentos oportunos o también, reforzar y potenciar aquello que ya se hace bien.

Dentro de los modelos que se preocupan de los procesos en el contexto de la Calidad Total está el "Modelo de Calidad y Excelencia" (EFQM) del que ya se ha hecho mención. Tal como lo señalan Medina y Medina [6], el Modelo EFQM abarca todos los aspectos importantes de una organización y se le puede considerar como "...un marco preceptivo, que no ata a las organizaciones que lo deseen utilizar..." [6] y enfatizan que favorece la cultura de las instituciones, lo que es fundamental, especialmente porque el modelo ha sido principalmente aplicado a instituciones de educación superior de un tamaño superior que el de la Universidad Mayor.

Existen diferentes investigaciones en torno a la aplicación del Modelo EFQM en diferentes niveles educativos. En la década pasada se hicieron investigaciones para aplicar el modelo en conjunto de universidades del Reino Unido, donde enfatizaron la metodología para la autoevaluación, proporcionando información relevante que permite deducir que se favorece la orientación hacía el estudiante por parte de las instituciones que lo aplican. Otros autores, dan cuenta de diferentes elementos que apoyan la gestión de calidad y que también forman parte del EFQM, como elementos centrales a ser considerados [4-8].

Entre los elementos que se destacan y que el modelo EFQM considera como relevante para ser efectivo en su aplicación, es que se requiere que se integren y alineen diferentes componentes de la organización, como lo son el planeamiento, el involucramiento del personal por medio de la conformación de equipos para abordar la autoevaluación e introducir cambios, y destaca la "performance" gerencial como un elemento transversal para que los cambios ocurran [8].

Durante las dos últimas décadas, varias adaptaciones del Modelo EFQM, donde se adaptaron conceptos para que fuera más comprensible su aplicación a la educación superior, por ejemplo, el concepto de "cliente" explicita se refiere a los estudiantes, sus padres y empleadores. Las numerosas investigaciones sobre la temática objeto de estudio plantean la necesidad de aplicar este modelo en diferentes 
instituciones educativas donde destacaron la importancia de la autoevaluación para la mejora continua. En la bibliografía especializada se señala la utilización para introducir mejoras en los análisis $\mathrm{y}$ verificaciones de efectividad en las instituciones.

\section{Mejoramiento y participación}

La denominada filosofía Kaizen, permite sustentar una metodología de autoevaluación que se centra en el mejoramiento continuo, comprometiendo la participación de los actores claves, en el caso de una universidad se puede mencionar desde las autoridades centrales hasta el personal administrativo y de servicios. Las organizaciones que trabajan bajo esta filosofía orientan el perfeccionamiento de los procesos para mejorar los resultados, pero lo interesante es que plantea que las mismas personas que conocen los procesos son las más indicadas para encontrar los problemas o áreas que requieren atención para la mejora [7].

La planificación estratégica, entendida como proceso que busca alcanzar las metas y objetivos en miras del cumplimiento de la misión y la excelencia, busca enmarcar el quehacer en un enfoque sistémico, donde convergen todos los ámbitos institucionales en búsqueda de la calidad [3-5].

Riveros [3] señala que "Dentro de las nuevas normas organizativas de la universidad no deja de tener importancia el tema de la participación" Lo cual es ratificado también por otros autores [4].

En todo proceso de autoevaluación es imprescindible el trabajo en equipo, también mencionado por [6-8] entre otros, el que debe estar presente en los procesos de mejora de la calidad del servicio; el aporte de cada individuo permite lograr los objetivos del conjunto, pues se potenciarán y complementarán a partir de sus conocimientos, habilidades, experiencias y perspectivas. El problema que plantea Lamaitre es que en Chile justamente la participación de los diferentes actores en los procesos de planificación y acreditación es escasa, especialmente porque se percibe como procesos entrampados en la burocracia, donde el sentido de lo que se hace y el propósito se ve con poca precisión [5-7].

Ese trabajo en equipo debe estar en función de la satisfacción del cliente, entendido este como el estudiante, su familia, los proveedores o el mismo personal que debe acudir a una unidad administrativa o de servicios para solicitar información o apoyo que le permitirá ser más efectivo en su tarea.

La satisfacción de las expectativas y de las necesidades de los usuarios claves constituye uno de los aspectos más importantes en cualquier proceso de gestión de la calidad y según diferentes autores se le considera uno de los factores de éxito más importante para las organizaciones. Por este motivo, es necesario medir la satisfacción de los usuarios claves y también ser capaces de establecer las medidas para introducir mejoras inmediatas ante la insatisfacción que se genere en un determinado momento [5].

\section{Modelos para la autoevaluación y el mejoramiento} Otro elemento que fundamenta este trabajo es el Análisis de Pareto y el Análisis Multicriterio. El primero, se refiere a un ordenamiento de diferentes factores relativos a un problema, los que se comparan de modo de identificar y enfocar aquello que es esencial de lo que es secundario, lo que permite una priorización de los problemas relacionados a la calidad de un servicio, y lo que es aún más importante, posibilita el diagnóstico de las causas para a su vez priorizar la solución de las mismas. El segundo, se basa en criterios explícitos para evaluar diferentes alternativas, siendo utilizado cuando un grupo de personas debe tomar una decisión que involucra diferentes aspectos, especialmente complejos o que generan conflictos, usualmente en el momento de seleccionar o buscar alternativas.

A partir de la revisión bibliográfica para conocer el estado del arte en materia de autoevaluación de unidades de gestión, se optó por utilizar como referente los criterios del "Modelo EFQM de Calidad y Excelencia" [4]. No es posible replicar todos los aspectos considerados en la génesis o en las actualizaciones, sino solo aspectos que subyacen de carácter conceptual, de modo de orientar la aplicabilidad en un proceso de autoevaluación, contextualizado a la Universidad Mayor, tal como lo hacen muchas organizaciones que lo utilizan. Sus características hacen que sea posible, especialmente los nueve conceptos que recogen los criterios que permitirán orientar el análisis interno de las unidades. Además de lo señalado se tomaron en cuenta otros aspectos presentes en las metodologías relacionadas a la calidad total [2]. 
$\mathrm{Al}$ respecto [4] señala que: "El origen empresarial del modelo origina ciertas dificultades no solo en lo que se refiere a la terminología utilizada...", sino que además hace referencia a que existen ciertas características en las diferentes organizaciones que deben ser tomadas en cuenta, como por ejemplo la organización interna, los estilos de dirección, las formas de gestionar, el tipo de tareas y usuarios, entre otras limitaciones. Algo similar ocurre con la matriz de cálculo sugerida, la que tiene un estándar alto, puesto que se usa como medio para premiar y rankear a las empresas, lo que hace que no siempre sea pertinente a los fines de otras organizaciones, como lo es en este caso.

Los aspectos revisados abordan diferentes dimensiones que deben ser consideradas en un proceso de autoevaluación de unidades administrativas como las de servicio de una organización universitaria, puesto que como todo proceso de autoevaluación este será realizado entre pares y por pares, lo que debe asegurar la participación de las personas que trabajan en la organización, con una mirada de apertura y flexibilidad de modo que se constituyan en agentes de cambio para tender al mejoramiento continuo, tal como lo plantea la filosofia Kaizen [7].

\section{El diseño metodológico}

En esta investigación se utilizó una metodología mixta, siendo el procedimiento secuencial el que respondía mejor al diseño, puesto que se partió con una metodología cualitativa a nivel exploratorio para continuar a partir de los primeros resultados, con una metodología cuantitativa, lo que posibilitó la profundización y generalización de los resultados [10].

La investigación se encuentra dentro de los estudios exploratorios y descriptivos. No se encontraron evidencias de desarrollos de instrumentos con estas características en las universidades chilenas. Según Hernández, los diseños de este tipo recolectan datos e información nueva de alguna área u objeto de estudio, como lo es en este caso, lo que permite aportar directamente con el cuerpo de conocimientos en la aplicación de mecanismos de aseguramiento de la calidad de instituciones de educación superior [11].

Este estudio se complementa con su carácter descriptivo, en cuanto buscó identificar ciertas características propias de las unidades en estudio, es decir, recolectar datos que dan cuenta de diferentes dimensiones o componentes del fenómeno foco de la investigación [11].

\section{Primera etapa del estudio}

En la fase exploratoria se realizaron entrevistas en profundidad a doce miembros de la organización, que tenían diferente formación profesional y jerarquía, los que formaban parte del universo. A partir de los resultados, se elaboraron los ítemes preliminares, los que fueron sometidos a consulta con un grupo de expertos, cuatro especialistas en aseguramiento de la calidad y gestión universitaria; un experto en calidad total externo a la institución de manera que con su juicio se pudiera llegar a la propuesta inicial y así construir el instrumento. Para su selección se identificaron criterios, entre los que se destacan: la antigüedad, el conocimiento de la institución, la experiencia en educación superior y en gestión universitaria, entre otros. Se invitaron varios expertos que siendo externos a la institución, tenían dominio y experiencia en el ámbito del aseguramiento de la calidad.

Con los resultados de esta etapa, se diseñó el instrumento y se hicieron los ajustes para determinar las adaptaciones a las dimensiones que tendrían, no solamente el instrumento en particular, sino que servirían de guía al sistema completo de autoevaluación para estas unidades, puesto que se detectó en esta etapa que se requeriría de material para la capacitación de los funcionarios y otros instrumentos para guiar la reflexión y la elaboración de planes de mejora.

El propósito del cuestionario de autoevaluación es servir de herramienta a las unidades o áreas de trabajo de apoyo a la gestión de la Universidad Mayor para hacer su proceso de autoevaluación, de manera sencilla y práctica, y constituirse en un diagnóstico del nivel de logro con respecto a los criterios que componen este modelo y aportar a la mejora continua. Se determinaron dos variables. La primera, relacionada a los Facilitadores del Proceso, es decir todo aquello que permite que una unidad provea del servicio o realice su quehacer. $\mathrm{La}$ segunda, se relacionó a los Resultados, es decir, qué se puede esperar que se realice o logre en una unidad de servicio o administrativa determinada. Ambas variables se operacionalizaron en criterios mucho más específicos y alrededor de esas definiciones 
se elaboraron los indicadores del instrumento de evaluación y permitieron, además, estructurar los temas de los Comités de Autoevaluación de las 36 unidades participantes.

\section{Comparación entre los criterios originales del EFQM y la adaptación para la Universidad Mayor (UM)}

A continuación, se hace una breve referencia de cómo, a partir de los conceptos originales que subyacen en el modelo del EFQM, se realizó la adaptación para la Universidad Mayor, tomando los resultados de la primera etapa del estudio. De esta forma se aseguró que el sistema y el instrumento de autoevaluación tuviera sentido en la cultura institucional.

\section{Concepto original EFQM: Liderazgo. Criterio para UM: Liderazgo.}

El concepto se mantiene y se orienta a cómo se desarrollan y se ponen en práctica la cultura y los valores institucionales, reflejados en la percepción que se tiene sobre el liderazgo, lo que es importante para contribuir al éxito institucional. Especialmente interesa detectar si se reconoce el liderazgo de algunos actores claves del gobierno universitario.

2. Concepto original EFQM: Política y Estrategia. Criterio para UM: Política y Estrategia.

Se mantiene el concepto y se orienta a cómo la unidad desarrolla su misión y visión y las pone en práctica por medio de una clara estrategia orientada hacia los distintos agentes y personas con quienes interactúa, siendo coherente con la misión institucional y los objetivos estratégicos.

\section{Concepto original: Personal Concepto para UM: Funcionarios}

Se adapta para acercar el término al lenguaje institucional. Se enfoca a cómo la unidad desarrolla, conduce y aprovecha el potencial de las personas, de forma individual y grupal, con el propósito de contribuir a la gestión de la unidad.

4. Concepto original: Colaboradores.

Concepto para UM: Alianzas y recursos.

Se adapta para acercar el término al lenguaje institucional. Se enfoca a cómo la unidad gestiona los recursos internos que requiere (espacios, equipos, materiales, información, conocimientos, recursos tecnológicos, entre otros) y también los externos a la unidad que se autoevalúa (proveedores, otros servicios de la institución, con otros organismos, entre otros), con el propósito de contribuir a la gestión de la unidad.

\section{Concepto original EFQM: Procesos Criterio para UM: Procesos Internos.}

Se explicita qué son procesos internos para facilitar la comprensión del criterio. Se enfoca a analizar algunos de los procesos de gestión más relevantes de la unidad que se autoevalúa, de modo de identificar cómo se desarrollan y mejoran con el fin de satisfacer las necesidades y expectativas de los diferentes usuarios mediante el servicio que se entrega y cómo también esto repercute en los estudiantes.

6. Concepto original EFQM: Resultados en clientes. Criterio para UM: Resultados en Usuarios Frecuentes.

Se adapta para utilizar la denominación de usuarios frecuentes y posibilitar que se enfoque en los que demandan un servicio o gestión de la unidad, sean internos o externos de la institución. El eje esta puesto en analizar los resultados o productos principales que logra la unidad con el servicio que brinda, reconociendo que la principal preocupación y objetivo diario es aportar a la formación de los estudiantes de las diferentes carreras y programas.

\section{Concepto original EFQM: Resultados en Personal \\ Criterio para UM: Resultados en Funcionarios.}

Se adapta. Se focaliza a analizar cómo la unidad consigue favorecer el desarrollo de las personas y su compromiso con la institución.

8. Concepto original: EFQM: Resultados en la Sociedad.

Criterio para UM: Resultados en la Comunidad Universitaria.

Se adapta y dado la realidad y el contexto institucional, se restringe el criterio a la comunidad interna de la organización. Se focaliza a detectar cómo la unidad consigue establecer relaciones con otras unidades 
internas y con el entorno, según corresponda como medio para potenciar su gestión y contribución a la UM en su conjunto.

9. Concepto original EFQM: Rendimiento. Criterio para UM: Resultados Claves.

Se cambia para responder a la heterogeneidad de las unidades, pero enfocado a valorar los principales servicios, acciones, procedimientos que tienen relación con el quehacer de la unidad que se autoevalúa y cómo logra sus objetivos, satisfaciendo las necesidades y expectativas relacionadas a su propio quehacer.

El instrumento fue elaborado a partir de las descripciones precedentes, desde la perspectiva de las unidades existentes e interesadas en la realización de la autoevaluación de cuestionarios anteriores utilizados por la Universidad Mayor y de aspectos contenidos relacionados a gestión y procedimientos habituales de las unidades administrativas y de servicios, para efectos de acreditación institucional. Inicialmente el instrumento quedó diseñado con 45 proposiciones después de la primera validación con juicios de expertos, quedó en las 51 que se llevaron a la evaluación con la muestra.

Se construyeron dos escalas tipo Likert, con valoraciones de puntaje tal como lo muestra la Tabla 1.

\section{Segunda etapa del estudio}

Se administró el instrumento con los 51 ítems, de manera on line y bajo una metodología cuantitativa se procedió a procesar los datos para verificar su validez y si era efectivamente posible aplicarlo. Uno de los desafíos de esta etapa era por una parte, lograr la mayor participación posible y adhesión con el sistema por parte de los funcionarios, de manera de sobrellevar las diferencias de formación -culturales y sociales- puesto que la muestra era muy heterogénea.

Esta muestra fue probabilística, quedando de un universo de 509 personas -376 de la sede Santiago y 133 de la sede Temuco- un total de 397, entre autoridades, jefaturas y personal sin cargo directivo. Fue una participación voluntaria y el total se agrupaba en 36 unidades diferentes (administrativas y de servicios). Posterior a la aplicación, se invalidaron cuestionarios al verificarse que no cumplían algunos de los requisitos establecidos previamente.

Se puede verificar que la muestra cumplió con un 99\% de confianza y el error muestral fue del $3 \%$.

Finalmente, el cuestionario quedó conformado por 58 ítems, lo que constituyó el instrumento definitivo y que incorporó los aportes de la etapa de evaluación con la muestra completa.

\section{RESULTADOS}

Con la valoración de los puntajes absolutos para cada ítem y de acuerdo a la escala correspondiente, se obtuvo un puntaje ponderado individual que representa el máximo (Puntaje máx. ponderado individual) que puede obtener dicho ítem. Esto lleva a obtener para cada criterio de autoevaluación un puntaje máximo ponderado, que consiste en multiplicar los valores individuales por el número de la muestra (n), teniendo entonces el puntaje que podría idealmente tener el instrumento en esa muestra y que representa una situación deseable de calidad. (Puntaje máx. ponderado). Esto se refleja en la ecuación (1):

$$
\begin{aligned}
& \text { Puntaje Max. ponderado }{ }_{\text {criterio }}= \\
& \text { SPje Max.ponderado indiv. }{ }^{*} n
\end{aligned}
$$

Tabla 1. Escala 1 de valoración.

\begin{tabular}{|l|c|c|c|}
\hline \multicolumn{1}{|c|}{ Criterio de evaluación } & $\begin{array}{c}\text { Valoración } \\
\text { (ptos.) }\end{array}$ & Criterio de evaluación & $\begin{array}{c}\text { Valoración } \\
\text { (ptos.) }\end{array}$ \\
\hline No aplica o no corresponde (N/C) & 0 & No tengo información suficiente para responder & 0 \\
\hline Ningún avance & 1 & No & 1 \\
\hline Cierto avance & 2 & Sí & 2 \\
\hline Avance significativo & 3 & & \\
\hline Objetivo logrado & 4 & & \\
\hline
\end{tabular}


Para obtener el porcentaje de logro de cada criterio, se multiplica el "puntaje obtenido" por cien, dividiendo el resultado por el "puntaje máximo ponderado". Esto se traduce en el grado de calidad alcanzado en el criterio. Tal como lo muestra la ecuación (2):

$$
\text { Logro }_{\text {criterio }(\%)}=\frac{(\text { Puntaje obtenido })}{\text { Puntaje máx. } \text { ponderado }} * 100
$$

Los criterios con sus ponderaciones y puntaje máximos ponderados para el instrumento en la aplicación piloto se presentan en las Tablas 2 y 3.

Considerando estas ponderaciones, el logro total (en porcentaje) alcanzado por la unidad o área de trabajo es el mismo ejercicio anterior, pero utilizando la sumatoria de los puntajes obtenidos de los nueve criterios de autoevaluación, multiplicados por cien y divididos por la sumatoria del puntaje máximo ponderado para el $\mathrm{n}$ definido en la muestra.

Para favorecer el diagnóstico y el análisis, el porcentaje de logro alcanzado por la unidad, el criterio de excelencia o el ítem dentro del criterio fue clasificado de la siguiente forma:

- $90 \%$ a $100 \%$ : Fortaleza de excelencia. Son todos aquellos elementos positivos que se diferencian y destacan de otros de igual clase. Pueden ser recursos que se controlan, capacidades y habilidades que se poseen o actividades que se desarrollan de manera destacada.

- $\quad 80 \%$ a 89\%: Fortaleza. Son aquellos elementos logrados que pueden ser aprovechados para favorecer el desarrollo óptimo de la institución. El logro se relaciona directamente con lo que se espera de la unidad, aunque siempre habrá aspectos susceptibles de ir evolucionado.

- $50 \%$ a 79\%: Fortaleza con posibilidades de mejora. El logro presenta elementos que, aun siendo alcanzados, son parciales, puesto que se debe buscar el desarrollo de otros aspectos de la categoría y justifica una preocupación sistemática orientada a la mejora.

- $0 \%$ a 49\%: Debilidad. Refleja que no existe el logro esperado, lo que representa un obstáculo o problema en el desarrollo de la institución. Pueden ser recursos de los que se carece, habilidades que no se poseen, actividades que no se desarrollan positivamente, etc. Cualquiera sea lo no alcanzado afectará el logro de la unidad y sus objetivos más estratégicos.

Esta escala resultó ser consistente con los criterios de evaluación utilizados y sus puntajes.

En la segunda etapa se realizó una aplicación a la muestra para posteriormente proceder a su validación estadística, lo que se presenta en la discusión de resultados. Las respuestas fueron anónimas y se dio un plazo de no más de una semana para responder.

Tabla 2. Ponderación de los criterios y los puntajes máximos ponderados para la Variable 1.

\begin{tabular}{|l|l|c|c|}
\hline $\begin{array}{c}\text { Variable 1 } \\
\text { Gestión }\end{array}$ & \multicolumn{1}{|c|}{ Facilitadores } & $\begin{array}{c}\text { Ponderación } \\
(\mathbf{\%})\end{array}$ & Ptjes. máx. ponderación (n=397) \\
\hline & Liderazgo & 10 & 1.032 \\
\hline & Política y Estrategia & 8 & 635 \\
\hline & Funcionarios & 9 & 715 \\
\hline & Alianzas y Recursos & 9 & 572 \\
\hline & Procesos Internos & 14 & 1.779 \\
\hline
\end{tabular}

Tabla 3. Ponderación de los criterios y los puntajes máximos ponderados para la Variable 2.

\begin{tabular}{|l|l|c|c|}
\hline $\begin{array}{c}\text { Variable 2 } \\
\text { Resultados del proceso }\end{array}$ & \multicolumn{1}{|c|}{ Facilitadores } & $\begin{array}{c}\text { Ponderación } \\
(\mathbf{\%})\end{array}$ & Ptjes. máx. ponderación (n=397) \\
\hline & Usuarios Frecuentes & 20 & 2.223 \\
\hline & Resultados Funcionarios & 9 & 1.000 \\
\hline & Comunidad Universitaria & 6 & 265 \\
\hline & Resultados Claves & 15 & 1.172 \\
\hline
\end{tabular}


El análisis estadístico se realizó con el software SPSS. La fiabilidad del cuestionario se valoró mediante el Alfa de Cronbach es un coeficiente que evalúa la consistencia interna, es decir, el grado en que las respuestas obtenidas por cada ítem se correlacionan con el resto del cuestionario [12].

En el contexto de la autoevaluación, la validación de la encuesta se debe considerar como parte de un trabajo más amplio puesto que esta es un elemento que sirve para recoger datos que son utilizados por los equipos de trabajo durante el proceso, el que no concluye con su sola aplicación o con la obtención de sus resultados.

La muestra se puede caracterizar de diferentes maneras, siendo una de ellas el período de trabajo antigüedad en la unidad administrativa o de servicios. Es así que la mayoría -representada por el 44,8\% se desempeñaba en ese lugar entre uno a tres años, seguido por los que se ubicaban en el rango de entre cuatro a seis años $(17,1 \%)$ : Los menores valores están representados por aquellos funcionarios que trabajan en la institución entre siete y diez años $(8,6 \%)$ y más de diez años $(14,1 \%)$.

Se debe indicar que la muestra se organizó en subgrupos según rectoría y vicerrectorias, lo que permitió realizar un período de capacitación respecto al modelo completo de autoevaluación y en particular a la aplicación del instrumento. Es así que la mayor parte de los participantes corresponden a la Vicerrectoría Académica y de Aseguramiento de la Calidad y a la de Administración y Finanzas, las que representan el $54 \%$ de la muestra. El resto se distribuyó entre funcionarios de Rectoría, la Vicerrectoría de Desarrollo y la Vicerrectoría Regional de Temuco, siendo una distribución pareja entre estas unidades académicas. Es decir, todas las unidades quedaron representadas.

El personal se dividió para efectos del análisis en direcciones, encargados o subordinados. Los subordinados representaron el mayor número de participantes $(75,1 \%)$, le siguieron los encargados $(20,2 \%)$ y, por último, los que ostentan cargos relacionados a Direcciones $(4,8 \%)$.

En el análisis de fiabilidad, los nueve criterios medidos reflejaron una alta consistencia interna, como se refleja en la Tabla 4.
Tabla 4. Alfa de Cronbach por criterio.

\begin{tabular}{|l|c|}
\hline \multicolumn{1}{|c|}{ Criterios } & Alfa de Conbrach \\
\hline Liderazgo & 0,8 \\
\hline Política y Estrategia & 0,8 \\
\hline Funcionarios & 0,7 \\
\hline Alianzas y Recursos & 0,8 \\
\hline Procesos Internos & 0,9 \\
\hline Usuarios Frecuentes & 0,8 \\
\hline Resultados Funcionarios & 0,9 \\
\hline Comunidad Universitaria & 0,6 \\
\hline Resultados Claves & 0,9 \\
\hline
\end{tabular}

Para estudios realizados en el contexto de las organizaciones, un coeficiente menor a 0,6 indica una baja confiabilidad. En el caso de esta encuesta el menor valor encontrado fue de 0,6 para el criterio comunidad universitaria. Se puede definir que los nueve criterios son confiables, aunque el de comunidad universitaria debe ser observado en siguientes aplicaciones.

Se trata de que los funcionarios realicen una autoevaluación del desempeño de su unidad, pero también detectar su propia percepción de la institución que acoge a esta unidad, de manera de favorecer el automejoramiento en miras de sentirse parte del mismo. Cuando en la organización la persona evalúa el desempeño de su unidad, su eficiencia y eficacia, en función de lo que justifica la existencia de la misma y, por tanto, de su propio trabajo, se puede esperar que se favorezca la toma de conciencia de los propósitos estratégicos de la institución.

Para determinar la metodología fue necesario validar el constructo del instrumento de evaluación, así como determinar las etapas del proceso, cómo se orientarían y se llevará a cabo la autoevaluación y la posterior construcción de los planes de mejora de las unidades que participaron en el estudio.

\section{CONCLUSIONES}

La encuesta resultó ser válida y confiable, evidenciando una consistencia interna coherente lo que se debe, en parte, a la consideración de las propias ponderaciones del modelo original como por las reiteradas validaciones que se hicieron en la primera etapa con los diferentes actores involucrados, lo que permitió que se ajustaran los 
ítemes, que no solamente respondían a una realidad y cultura institucional, sino que tenían un lenguaje que resultó ser cercano y directo y de uso regular al interior de la universidad.

Una de las ventajas es que se pudo contar para la autoevaluación institucional con un instrumento que fue acogido por las diferentes unidades. La limitación es que se requiere de tiempo posterior para hacer las reuniones con todos los funcionarios y elaborar los planes de mejora y no siempre existe la disponibilidad.

Algunas recomendaciones que surgen del trabajo realizado son las siguientes:

Se requiere del compromiso de las autoridades de la institución para la aplicación de este instrumento, como una forma de asegurar que el proceso completo se lleve a cabo y no quede solo como la obtención de unos resultados por la aplicación del mismo. Se trata de abrir espacios de participación a todos los funcionarios.

Es necesario incluir la capacitación de todos los funcionarios antes del uso del instrumento, así como a las personas líderes de las unidades, estos últimos son claves para favorecer la participación. Introducir la cultura de la evaluación en el área administrativa y de servicios es una tarea importante para mejorar la calidad del servicio que se presta a los estudiantes.

Este instrumento es un eslabón de una cadena más amplía, puesto que el aseguramiento de la calidad requiere de los espacios de reflexión y análisis de los equipos de trabajo.

El instrumento considera la opinión y voz de los participantes, lo que fue valorado. Esta información es un insumo relevante que debe ser procesado con métodos cualitativos, puesto que complementa los análisis cuantitativos y posibilita profundizar o buscar respuestas a ciertas interrogantes o situaciones.

Finalmente, se puede decir que el instrumento se utilizó para el proceso de autoevaluación realizado el año 2015, tanto internacional y nacional, fue valorado por los pares chilenos y extranjeros porque aportaba en un espacio que en términos generales, es menos considerado en estos procesos de evaluación: los actores de las unidades administrativas y de servicios de la institución universitaria. Este instrumento validado es replicable en otras instituciones de educación superior.

\section{REFERENCIAS}

[1] A. Hax y J.J. Ugart. "Hacía la Gran Universidad Chilena. Un modelo de trasformación estratégica". Ediciones Universidad Católica de Chile. Santiago, Chile, pp. 108. 2014. ISBN: 978-956-14-1464-8.

[2] J. Cortadellas, C. García de Elias, S. Roca, F. Solá y J. Vilalta. "Guía de Evaluación de Servicios. Plan Nacional de Evaluación de la Calidad de las Universidades, Ministerio de Educación, Cultura y Deporte". Secretaría General Técnica. España. Fecha de consulta: 12 diciembre 2013. URL: http://www.ugr. es/ facmed/calidad/guiaservicios.pdf

[3] L. Riveros. "La Universidad Chilena. Los Efectos de una Mandrágora contemporánea", pp. 41. Editorial Occidente S.A. Santiago, Chile. 2013.

[4] J.A. Maderuelo. "Gestión de la Calidad Total: El Modelo EFQM de excelencia". Revista Medifam. Vol. $12 \mathrm{~N}^{\mathrm{o}}$ 10, pp. 638. Madrid, España. Diciembre 2012. Fecha de consulta: 19 noviembre 2013. URL: http://scielo.isciii.es/scielo.php?pid=s113157682002001000004\&script=sci_arttext

[5] A. Bernasconi (Editor). "La Educación Superior en Chile. Trasformación, Desarrollo y Crisis". Cap. VI. "Aseguramiento de la Calidad: Una Política y sus Circunstancias". De M.J. Lemaitre. Colección Estudios en Educación. Ediciones Universidad Católica de Chile. Santiago, Chile. ISBN: 978-956-14-1492-1.

[6] M. Medina y E. Medina. "La formación para la gestión de la calidad en Servicios Sociales. Cómo plantearla en el Grado en Trabajo Social". Revista Interuniversitaria de Formación del Profesorado, pp. 125-143. Universidad de Zaragoza, España. Fecha de consulta: 19 julio 2014. URL: http://www. redalyc.org/pdf/274/27419173009.pdf

[7] M. Suárez. "El Kaizen: La filosofía de mejora continua e innovación incremental detrás de la administración por calidad total”, pp. 49. 
Panorama Editorial. Ciudad de México, México. ISBN: 978-956-14-1492-1.

[8] P. Dietric, P. Eskerod, D. Dalcher and B. Sandhawalia. "The Dynamics of Collaboration in Multipartner Projects". Project Management Journal. Vol. $41 \mathrm{~N}^{\circ} 4$, pp. 59-78. September, 2010. United States. Fecha de consulta: 27 enero 2014. URL: http://dx.doi.org/10.1002/pmj.20194

[9] C. Andrade y N. Labarca. "Fundamentación Teórica de los modelos de gestión de calidad en el servicio de información en instituciones universitarias". Revista Omnia. Vol. 17, pp. 82-95. Enero-abril 2011. Fecha de consulta: 5 Diciembre 2013. URL: http:// www.produccioncientíficaluz.org/ index. php/omnia/article/view/7346/7335
[10] Z. Pereira. "Los diseños de Método mixto en la investigación en educación: Una experiencia concreta". Revista Electrónica Educare. Vol. XV No 1, pp. 15-29. Junio 2011. Fecha de consulta: 12 julio 2014. URL: http://www.revistas.una.ac.cr/index. php/EDUCARE/article/viewFile/867/793

[11] R. Hernández, C. Fernández y P. Baptista. "Metodología de la Investigación". Mc Graw-Hill Interamericana. $6^{\mathrm{a}}$ edición. Ciudad de México. México. 2014. ISBN: 14-562-239-6

[12] R. Sierra. "Técnicas de Investigación Social. Teorías y Ejercicios”. Paraninfo. 14 Edición, $4^{\text {a }}$ Reimpresión. Madrid, España. 2007. ISBN: 978-84-283-2429-8. 